\title{
Primary Malignant Melanoma of the Oral Mucosa: Report of an Unusual Case
}

\author{
Btissaam. Belhoucha ${ }^{1, *}$, Zahra Essaadi ${ }^{2}$, Othmane Benhommad ${ }^{1}$, Youssef. Rochdi ${ }^{1}$, Hassan. Nouri ${ }^{1}$, \\ lahcen. Aderdour ${ }^{1}, M_{\text {Khouchani }}{ }^{2}$, Abdelaziz. Raji ${ }^{1}$ \\ ${ }^{1}$ Department of ENT, CHU Med VI, Marrakech 2360, Morocco \\ ${ }^{2}$ Department of Oncology and radiotherapy, CHU Med VI, Marrakech, Morocco \\ *Corresponding author: btissambelhoucha@gmail.com
}

Received August 11, 2014; Revised August 18, 2014; Accepted August 21, 2014

\begin{abstract}
Primary oral malignant melanoma is an extremely rare tumor, representing $0.2 \%$ to $8 \%$ of all melanomas; accounting for $0.5 \%$ of all oral malignancies The prognosis for a patient with oral melanoma is dismal, with a 5-year survival rate of $5 \%$ to $20 \%$. A case is reported of a malignant melanoma in the mouth of a 81 year old male. The patient had a history of a continuously growing exophytic hyperplastic mass of a dark color. Histological examination revealed a melanin-producing tumour. Surgery was not offered to our elderly and medically compromised patient. Instead he received radiation therapy as the primary treatment and immunotherapy as the adjuvant treatment.
\end{abstract}

Keywords: malignant melanoma, oral mucosa, radiation therapy, prognosis

Cite This Article: Btissaam. Belhoucha, Zahra Essaadi, Othmane Benhommad, Youssef. Rochdi, Hassan. Nouri, lahcen. Aderdour, M Khouchani, and Abdelaziz. Raji, "Primary Malignant Melanoma of the Oral Mucosa: Report of an Unusual Case.” International Journal of Dental Sciences and Research, vol. 2, no. 4 (2014): 103105. doi: 10.12691/ijdsr-2-4-8.

\section{Introduction}

Primary oral malignant melanoma is an extremely rare tumor, representing $0.2 \%$ to $8 \%$ of all melanomas, arising from the uncontrolled growth of melanocytes found in the basal layer of the oral mucous membranes; accounting for $0.5 \%$ of all oral malignancies $[1,2,4]$. The most frequent oral sites of occurrence are the palate and the maxillary gingiva. The clinical presentation of this condition can vary widely, from a typically pigmented macular or proliferative lesion, to a non-pigmented, soft vascular tumour, single or multiple, primary or metastatic, and only biopsy can establish the diagnosis. The prognosis for a patient with oral melanoma is dismal, with a 5-year survival rate of $5 \%$ to $20 \%$. The purpose of this article is to present a case of oral melanoma which was managed in our institution. A review of the literature is also presented.

\section{Case Report}

A 81 year old male was referred to the University Clinic of ENT Surgery in February 2013 with chief complaint of pain and swelling in the left gums.

The patient gave a history of smoking and alcohol use. Medical history was noncontributory.

He noticed a dark exophytic hyperplastic mass on the gingivae of the middle portion of mandibular anterior region, which bled during mastication.
The clinical examination revealed a large mass of $4 \times 2$ $\mathrm{cm}$ in dimension on the lingual aspect of mandibular anterior region and interdental gingival, extending from the mandibular right lateral incisor up to the left second premolar. Computed tomogram (CT) revealed a lesion measuring $4 \times 2 \mathrm{~cm}$, extending from right mandibular lateral incisor up to the left second premolar. There was erosion of the anterior cortex of the left body of mandible to the symphyseal region.

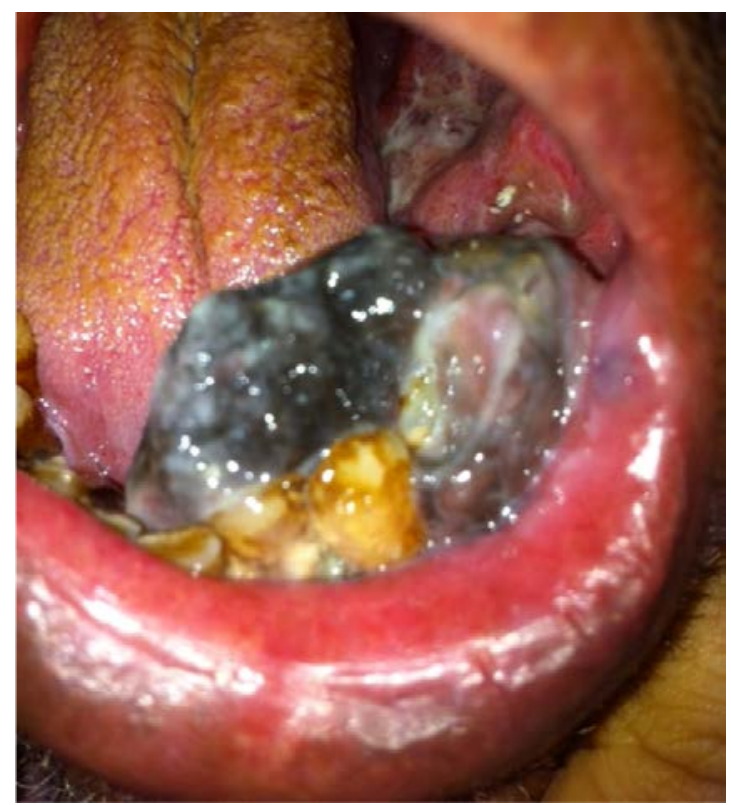

Figure 1. croscopic appearance of the tumor 


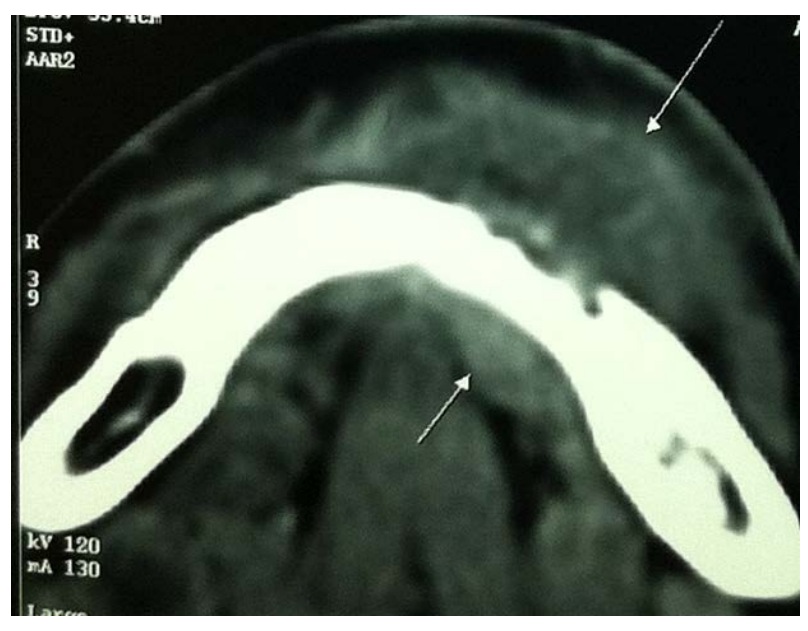

Figure 2. CT scan cut objectifying tumor process with bone lysis

Cervical examination showed neck node, measuring 4 cm. A CT examination of the neck, chest and abdomen, bone scanning, and ultrasounds of the liver and kidneys revealed enlarged neck nodes on CT measuring $4 \mathrm{~cm}$ and a liver metastases.

Therefore, incisional biopsy of the mass was performed. Routine microscopic examination of the specimen showed a melanin producing tumour, consisting of atypical irregularly elongated spindle- and oval-shaped melanocytes, xhibiting uniformly dark, enlarged and irregular nuclei. Immunohistochemical examinations was positif for HMB-45 and S-100 protein.

According to UICC (Union Internationale Contre le Cancer) staging, the patient was declared to be of "stage III," as he initially presented with enlarged neck nodes on CT and a liver metastases [3].

Surgery was not offered to our elderly and medically compromised patient. Instead he received radiation therapy as the primary treatment and immunotherapy as the adjuvant treatment.

Nine months after the adjuvant treatment. Our patient developed, in addition of liver metastases, pulmonary metastases which were the main cause of death secondary to respiratory failure and subsequent cardiopulmonary collapse (Figure 3).

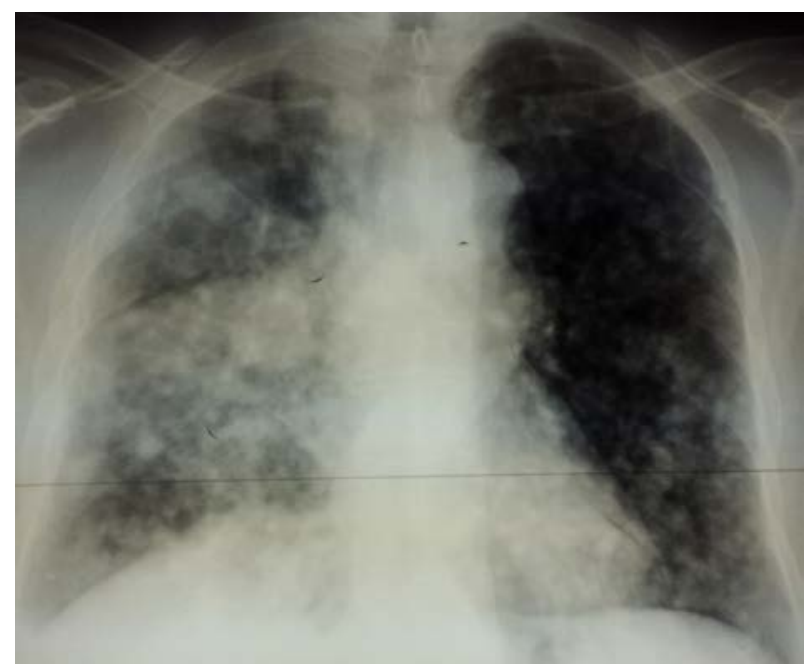

Figure 3. radiological appearance of lung metastasis
The most frequent head and neck site of occurrence of mucosal melanoma is the conjunctiva, followed by the upper respiratory tract $[1,2,3]$. Mucosal melanoma of the oral cavity is a rare malignancy, representing $0.2 \%$ to $8 \%$ of all melanomas. IT may be primary or metastatic from other locations in the body $[1,2,4]$. According to Greene et al [9], the criteria for the diagnosis of a primary oral melanoma are the following: 1) demonstration of clinical and microscopic tumor in the oral mucosa, 2) presence of junctional activity in the lesion and 3) inability to show any other primary site. Our patients fulfilled of these criteria [1,4].

The etiology of oral melanoma is unknown, and it has no known predisposing factors. Symptoms of oral melanomas vary and include a bleeding lump and, rarely, pain. The diagnosis is often unfortunately delayed until symptoms resulting from ulceration, growth, or bleeding are noted $[1,2,3,4]$.

In this case, the patient had a history of a continuously growing exophytic hyperplastic mass of a dark color.

Intra-oral naevi are uncommon lesions and, although their malignant transformation is less well supported than those of the skin, it is accepted that malignant melanoma may arise from neoplastic transformation of either melanocytes or naevus cells $[4,5,6]$. According to some studies, a history of preexisting melanosis can be found in about one third of all patients with oral melanoma [1].

Unlike their skin counterparts, mucosal melanomas cannot be classified into the Cutaneus melanomas categories due to different clinical and histologic characteristics.

Patients may be staged according to the American Joint Committee on Cancer. Alternatively, tumors may be stratified as follows According to UICC (Union Internationale Contre le Cancer) staging: stage I, confined to the primary site; stage II, positive cervical lymph nodes; and stage III, distant metastases [3,6].

The oral melanoma is more aggressive and the abundant blood supply of the oral cavity may permit blood vessel invasion and hematogenous dissemination early in the course of the disease $[3,5,6]$.

They usually occur on the hard palate and less frequently in the buccal and labial mucosa, gingiva and alveolar ridge, and may present as small, elevated papules, frequently nonpigmented up to 20 per cent $[4,5]$.

This fact may make it difficult to clinically appreciate malignant changes and the development of underlying melanoma in such lesions. Thus only biopsy can identify those with malignant potential. Fine needle aspiration or exfoliative cytology of primary pigmented lesions is contraindicated $[3,6]$.

The most common sites of metastasis are lung, bone, brain, and liver, with widespread involvement occurring in advanced disease $[3,4]$. In this case, the patient had a liver and a lung metastasis.

Microscopically, the tumor cells present themselves as densely packed, large epithelioid cells with eosinophilic cytoplasm. The melanin pigment may be abundant, but may be sparse or even absent at the light microscopic level. immunohistochemical stains are of significant help. Useful markers include S-100 protein, gp 100 (HMB-45), and Mart-1 (Melan-A) [4,6,7].

It has always been suggested that cutting into malignant neoplasm during incisional biopsy could result

\section{Discussion}


in accidental dissemination of malignant cells within adjacent tissues or blood or lymphatic stream with subsequent risk of local recurrence or regional or distant metastasis. But Lederman and Sober did find no correlation in patient's prognosis with incisional and excisional biopsies [4,8].

The treatment of oral malignant melanoma is still controversial and there is no census regarding the best therapeutic approach.It includes surgical resection with or without neck dissection, immunochemotherapy, and radiation therapy $[1,4,5,6]$. Surgery remains the most effective treatment for malignant melanoma and aggressive surgical control of local disease may result in prolonged disease-free survival $[1,4]$.

The current guidelines for the surgical management of primary cutaneous melanoma recommend a diagnostic excisional biopsy of the lesion followed by a wide local excision where the diagnosis is proved; with a surgical margin of 20 to $50 \mathrm{~mm}$. this kind of resection is not always possible for oral malignant melanoma. Excision of the primary lesion, preferably using an intraoral approach and involving at least $1.5 \mathrm{~cm}$ of healthy tissue, is recommended [3,4].

The issue of whether it is necessary to perform a neck lymph node dissection remains controversial. [1,6] Neck dissection should be reserved for cases with preoperatively confirmed lymph node metastases [3].

Radiation therapy is applied mainly on patients unable to undergo surgical treatment or as an adjuvant to surgery if poor prognostic pathologic features are present, such as multiple positive nodes, or extranodal spread of metastastic melanoma, even though oral melanomas are regarded as poorly radiosensitive [3].

Immunochemotherapy is used as an adjuvant to surgical resection. Even though the effectiveness of this therapie is mostly unknown [3].

Despite the improvement of surgical techniques and the introduction of new chemotherapeutic agents, prognosis of this malignancy remains poor. The dismal prognosis of the condition may be attributed to incomplete resection due to anatomical limitations in association with its long and silent course [4]. The prognosis for a patient with oral melanoma is dismal, with a 5 -year survival rate of $5 \%$ to $20 \%$ [1,4]. Recurrences may occur even 10-15 years after primary therapy. Distant metastases to the lungs, brain, liver, and bones are frequently observed [3].

On the other hand, good results have been reported and it has been emphasized that the disease is potentially curable if diagnosed and treated at an early stage $[4,5,8]$.

\section{Conclusion}

The occurrence of primary oral melanoma is very rare. Estimated at between 0.2 and 8 per cent of all melanomas. The prognosis of primary oral melanoma remains poor despite adequate locoregional control of the disease. The generally advanced stage of the tumor at initial diagnosis leads to a poorer survival of patients with mucosal melanomas as compared with patients with cutaneous melanomas. Tumor size and metastases are related to the prognosis of the disease. Early detection, therefore, is important. All oral pigmented lesions that are not clinically diagnostic should be biopsied $[1,2,3]$.

\section{Conflict of Interest Statement}

None.

\section{Funding}

None.

\section{References}

[1] Rapidis A, Apostolidis C, Vilos G, Valsamis S. Primary malignant melanoma of the oral mucosa. J Oral Maxillofac Surg. 2003; 61: 1132-9.

[2] Dimitra kopoulos, Lazaridis, A. Skordalaki. Primary malignant melanoma of the oral cavity. Report of an unusual case Australian Dental Journal 1998; 43: (6): 379-81.

[3] Neeraj Sharma. Primary Oral Malignant Melanoma: Two Case Reports and Review of Literature. Case Reports in Dentistry. Volume 2012, Article ID 975358, 5 pages.

[4] Bhullar $\mathrm{RP}^{1}$ et al. Primary melanoma of oral mucosa: A case report and review of literature; Dent Res J (Isfahan). 2012 May; 9(3): 353-6.

[5] Chang A, Karnell L, Menck H. The National Cancer Data Base report on cutaneous and noncutaneous melanoma: a summary of 84,836 cases from the past decade. The American College of Surgeons Commission on Cancer and the American Cancer Society. Cancer. 1998; 83: 1664-78.

[6] Hicks M, Flaitz C. Oral mucosal melanoma: epidemiology and pathobiology. Oral Oncol. 2000; 36: 152-69.

[7] M. Magremanne, C. Vervaet; Mélanome de la cavité buccale ; Revue de Stomatologie et de Chirurgie Maxillo-faciale, Volume 109, Issue 3, June 2008, Pages 175-177.

[8] Meleti M, Leemans CR, Mooi WJ, Vescovi P, van der Waal I. Oral malignant melanoma: A review of the literature. Oral Oncol. 2007; 43: 116-21.

[9] Greene GW, Haynes JW, Dozier M, et al: Primary malignant melanoma of the oral mucosa. Oral Surg Oral Med Oral Pathol 6:1435, 1953. 